\title{
IceCube: A Kilometer-Scale Neutrino Observatory at the South Pole
}

\author{
Francis Halzen \\ Physics Department, University of Wisconsin, Madison, WI 53706, USA
}

\begin{abstract}
Solving the century-old puzzle of how and where cosmic rays are accelerated mostly drives the design of high-energy neutrino telescopes. It calls, along with a diversity of science goals reaching particle physics, astrophysics and cosmology, for the construction of a kilometer-scale neutrino detector. This led to the IceCube concept to transform a kilometer cube of transparent Antarctic Ice, one mile below the South Pole, into a neutrino telescope.
\end{abstract}

Whereas it has been realized for many decades that the case for neutrino astronomy is compelling (Gaisser, Halzen \& Stanev 1995), the real challenge has been to develop a reliable, expandable and affordable detector technology to build the kilometer-scale telescopes required. Conceptually, the technique is simple. In the case of a high-energy muon neutrino, for instance, the neutrino interacts with a hydrogen or oxygen nucleus in deep ocean water and produces a muon travelling in nearly the same direction as the neutrino. The blue Cerenkov light emitted along the muon's kilometer-long trajectory is detected by strings of photomultiplier tubes deployed at depth shielded from cosmic radiation. Although IceCube can identify the secondaries produced by neutrinos of all flavors, muons with interaction lengths exceeding $10 \mathrm{~km}$ at the highest energies can be collected far outside the detector and therefore the $\nu_{\mu}$ effective detector volume far exceeds the volume instrumented.

Elsewhere in these proceedings we have presented the case for kilometerscale neutrino observatories (Halzen 2003). The first first-generation telescope, AMANDA II, is approaching an integrated flux sensitivity to $\mathrm{TeV}-\mathrm{EeV}$ neutrinos of $\sim 0.05 \mathrm{~km}^{2}$ year. The instrument is sensitive to neutrino fluxes roughly equal to those emitted by the observed $\mathrm{TeV}$ gamma ray sources. It is too early to conclude whether first generation telescopes will discover sources of cosmic neutrinos, or whether it takes kilometer-scale observatories to see neutrinos associated with the enigmatic cosmic rays as anticipated by theoretical estimates. We do already know that it will take much larger detectors such as IceCube to study any sources that AMANDA II may discover.

IceCube (Wissing 2003) will consist of 80 kilometer-length strings, each instrumented with 60 10-inch photomultipliers spaced by $17 \mathrm{~m}$. The deepest module is $2.4 \mathrm{~km}$ below the surface. The strings are arranged at the apexes of equilateral triangles $125 \mathrm{~m}$ on a side. The instrumented (not effective!) detector volume is a cubic kilometer. A surface air shower detector, IceTop, consisting of 160 Auger-style Cerenkov detectors deployed over $1 \mathrm{~km}^{2}$ above IceCube, augments the deep-ice component by providing a tool for calibration, background rejection and air-shower physics, as illustrated in the figure. 


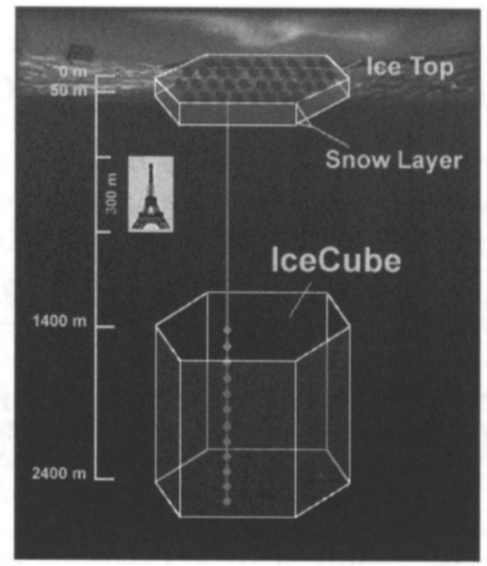

The transmission of analogue photomultiplier signals from the deep ice to the surface, used in AMANDA, has been abandoned. The photomultiplier signals will be captured and digitized inside the optical module. The digitized signals are given a global time stamp with a precision of $<10 \mathrm{~ns}$ and transmitted to the surface. The digital messages are sent to a string processor, a global event trigger and an event builder.

Construction of the detector is expected to commence in the Austral summer of 2004/2005 and continue for 6 years, possibly less. The growing detector will take data during construction, with each string coming online within days of deployment. The data streams of IceCube, and AMANDA II, embedded inside IceCube, will be merged off-line using GPS time stamps.

IceCube will offer great advantages over AMANDA II beyond its larger size: it will have a higher efficiency and superior angular resolution in reconstructing tracks, map showers from electron- and tau-neutrinos (events where both the production and decay of a $\tau$ produced by a $\nu_{\tau}$ can be identified) and, most importantly, measure neutrino energy. Simulations, backed by AMANDA data, indicate that the direction of muons can be determined with sub-degree accuracy and their energy measured to better than $30 \%$ in the logarithm of the energy. The direction of showers will be reconstructed to better than $10^{\circ}$ above $10 \mathrm{TeV}$ and the response in energy is linear and better than $20 \%$. Energy resolution is critical because, once one establishes that the energy exceeds $1 \mathrm{PeV}$, there is no atmospheric muon or neutrino background in a kilometer-square detector and full sky coverage of the telescope is achieved. The background counting rate of IceCube signals is expected to be less than $0.5 \mathrm{kHz}$ per optical sensor. In this low background environment, IceCube can detect the excess of anti- $\nu_{e}$ events from a galactic supernova.

\section{References}

Gaisser, T. K., Halzen, F. and Stanev, T. 1995,

Halzen, F. 2003, these proceedings

Wissing, H. 2003, $\mathrm{PhD}$ thesis (Humbold University, Berlin); Nucl. Inst. Meth. (to be published) and http://icecube.wisc.edu/science/sci-tech-docs/ 